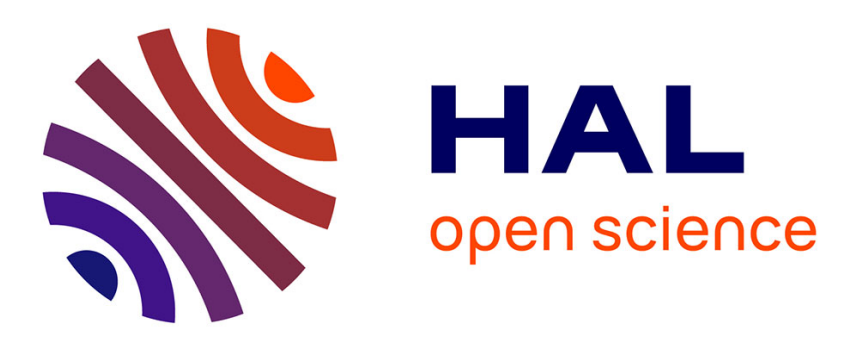

\title{
Formation of exciton rings and localized bright spots in coupled semiconductor quantum wells
}

\author{
S. V. Andreev
}

\section{To cite this version:}

S. V. Andreev. Formation of exciton rings and localized bright spots in coupled semiconductor quantum wells. Physical Review B: Condensed Matter and Materials Physics (1998-2015), 2016, 94 (16), pp.165308 (1-6). 10.1103/PhysRevB.94.165308 . hal-01397329

\section{HAL Id: hal-01397329 \\ https://hal.science/hal-01397329}

Submitted on 15 Nov 2016

HAL is a multi-disciplinary open access archive for the deposit and dissemination of scientific research documents, whether they are published or not. The documents may come from teaching and research institutions in France or abroad, or from public or private research centers.
L'archive ouverte pluridisciplinaire HAL, est destinée au dépôt et à la diffusion de documents scientifiques de niveau recherche, publiés ou non, émanant des établissements d'enseignement et de recherche français ou étrangers, des laboratoires publics ou privés.

\section{다(1)(2)}

Distributed under a Creative Commons Attribution - ShareAlikel 4.0 International 


\title{
Formation of exciton rings and localized bright spots in coupled semiconductor quantum wells
}

\author{
S. V. Andreev* \\ ITMO University, St. Petersburg 197101, Russia; \\ CNRS, LPTMS, Universite Paris Sud, UMR8626, 91405 Orsay, France; \\ and University of Bordeaux, LOMA UMR-CNRS 5798, F-33405 Talence Cedex, France
}

\begin{abstract}
We consider indirect excitons generated at the ring-shaped boundaries between electron- and hole-rich regions in semiconductor quantum wells (QW's). We show theoretically that the in-plane translational motion of the excitons is confined in the radial direction. The confinement potential results from the electrostatic interaction of the exciton dipole moment with the in-plane electric field induced at the boundary by the macroscopic charge separation. Our results directly apply to the external ring and the localized bright spots (LBS) observed in the photoluminescense (PL) pattern of indirect excitons.
\end{abstract}

\section{INTRODUCTION}

The observation of the external ring [1,2] and localized bright spots (LBS) $[1,3,4]$ in PL patterns of indirect excitons in biased semiconductor QW's has attracted much attention both on the experimental [5-8] and the theoretical [9-11] sides during the past 14 years. All these features were shown to originate from the reaction-diffusion dynamics of electrons and holes. The electrons are injected in the nearest to the positive lead QW layer by the current filaments due to the applied gate voltage. In the absence of photoexcitation, these electrons fill uniformly the layer plane. A focused laser is then used to excite electron-hole pairs in the surrounding barriers. Holes are captured efficiently by the QW layer close to the negative lead and form a lake of a positive charge around the excitation spot [12]. Electrons from the current filaments going through the lake bind with the holes to form excitonic ensembles seen as LBS rings in the PL pattern. The interface between the hole lake and the outer unperturbed electron sea is seen as the external PL ring.

The external and LBS rings are sources of cold exciton gases. Spontaneous onset of extended spatial coherence $[13,14]$, polarization textures [16], and macroscopic ordering [1] in the PL from these rings have been observed. Theoretical interpretation of these phenomena goes beyond the classical reaction-diffusion model and suggests emergence of novel quantum phases of excitons, in which the interplay between the dipolar interaction and spin degree of freedom can be studied at the macroscopic level [15-20].

Until now it has been assumed that, after an exciton has been created, it propagates out from the charge interface under combined action of diffusion and repulsive electrostatic interaction with other excitons. Careful analysis of the available experimental data indicates, however, that the assumption of free propagation of a single exciton in the radial direction should be revised. As can be inferred from the exciton PL patterns, the clouds squeeze (reduce the width) with lowering of temperature $[1,4]$. This experimental fact raises doubts on the free radial expansion picture, notably at low temperatures, where the exciton motion was argued to become ballistic

\footnotetext{
*serguei.andreev@u-psud.fr
}

$[16,17,19]$. Indeed, in contrast to the width of a cloud, the exciton coherence length increases when the temperature is lowered [14].

Here, we argue that in-plane translational motion of indirect excitons generated at the rings is confined in the radial direction. The confinement originates from electrostatic interaction of the exciton dipole moment with in-plane electric field induced by the macroscopic charge separation. The field tilts the dipoles, thus polarizing excitons in the structure plane. Polarized excitons seek for the region where the in-plane field is higher, i.e., the region of the charge interface. This way, we show that the external and LBS rings represent trapped exciton clouds.

Our argument provides a natural explanation to a whole series of experimental facts. First, it explains the aforementioned squeezing of the clouds when the temperature is reduced. Secondly, it allows us to reproduce the excitation power versus temperature (PT) phase diagram of the external ring reported in Ref. [1] (and, simultaneously, in Ref. [2]). Furthermore, it provides understanding to the "Mexican hat" spatial profile of the exciton blueshift measured in the vicinity of an LBS [3]. In fact, this observation had initially prompted a phenomenological interpretation of LBS's as trapped exciton clouds $[3,4]$. The bump in the central part of the energy profile has been explained as due to stimulated flux of excitons towards the center of the trap. This hypothesis is further supported by the experimentally observed universality in thermodynamic behavior of cold exciton gases: different LBS's containing strongly different number of excitons, develop the extended coherence and polarization textures all at the same critical temperature. This peculiar phenomenon was shown to be the distinct feature of two-dimensional Bose-Einstein condensation in an ensemble of harmonically trapped gases maintained at the same chemical potential [21,22].

The paper is organized as follows. In Sec. II, we consider interaction of a dipolar exciton with an in-plane field and obtain the formula for the exciton potential energy due to this interaction. To calculate the spatial distribution of the field (exciton potential energy), one needs to know distributions of electron and hole densities in charged regions. These are introduced in Sec. III following Ref. [9]. In Sec. IV, we calculate the electric field in the vicinity of the electron-hole interface. Section $\mathrm{V}$ is devoted to discussions and comparison 


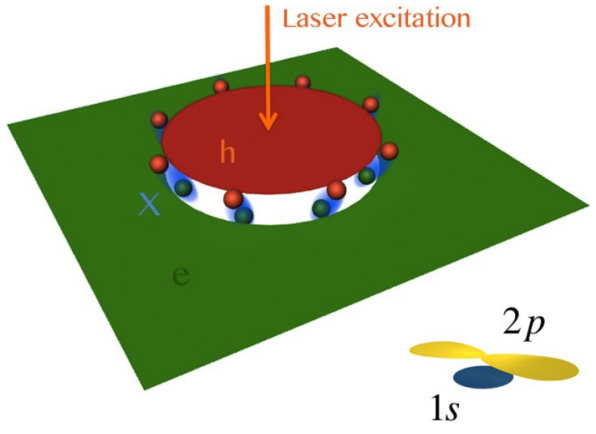

FIG. 1. The macroscipic charge separation induces an in-plane electric field which hybridizes $1 s$ and $2 p$ states of an indirect exciton. As a consequence, the excitons become polarized along the field. Classically, one can say that the field tilts the exciton dipole moment. Polarized excitons will seek for the regions where the induced electric field is stronger and, therefore, will become localized at the ringshaped boundary.

of our results with the available experimental data. We conclude this section by showing that our model of an LBS provides an adequate basis for theoretical interpretation of the coherence [14] and polarization [16] textures as well.

\section{TRAPPING MECHANISM}

Since the first experiments on cold exciton gases in QW's several trapping configurations have been proposed and realized. These include strain-induced traps [23-26], traps created by laser-induced interdiffusion [27], magnetic traps [28], laser-induced traps [29], and a large variety of proposals which rely on the electrostatic interaction of the exciton dipole moment with collinear electric field $E_{z}$ ( $z$ is the structure growth direction) [30-33]. In the latter case, the interaction energy is given by $\varepsilon(x, y)=-e d E_{z}(x, y)$, showing that the excitons will seek for the regions in the $(x, y)$ plane where the electric field is stronger.

In our case, the external transverse electric field applied to a bilayer QW structure is roughly uniform in the structure plane, so that no artificial potential landscapes for excitons is created. An indirect exciton generated at the ring has been assumed to propagate out from the source as a free particle (if one neglects the exciton-exciton interaction). We are going to show that at the carrier densities achieved in this kind of experiment the radial motion of excitons is confined, i.e., excitons are localized at the ring. The microscopic mechanism of this confinement is detailed below.

As we have already mentioned, the exciton ring appears at the boundary between the internal hole-rich region and the external electron-rich region. Thus one can expect the existence of in-plane electric field $\boldsymbol{E}_{r}(r, \theta)$ in the vicinity of the ring oriented everywhere outwards the center, along the radii. In the ground state an exciton does not interact with this field, since the relative two-dimensional motion of the electron-hole pair is characterized by a symmetric wave function. However, the interaction is already possible in the first order of the perturbation theory due to admixing of the $2 p$-exciton state. Classically, the electric field "stretches" the exciton, tilting its dipole moment (Fig. 1). As in the case of the artificially created potential profiles discussed above, interaction of an in-plane component of the exciton dipole moment with the built-in electric field at the boundary results in appearance of an electrostatic trap for the exciton in the radial direction, which localizes excitons at the ring.

The corresponding interaction energy can be estimated following the general rules of quantum mechanics. For that purpose, let us consider the quantum-mechanical problem of the relative in-plane motion of an electron and a hole confined to different QW's (or to diffrent edges of a wide single QW) and binded into an indirect exciton. The relative e-h motion is described by the Hamiltonian

$$
\mathcal{H}_{0}^{X}=-\frac{\hbar^{2}}{2 \mu}\left(\frac{\partial^{2}}{\partial \rho^{2}}+\frac{1}{\rho} \frac{\partial}{\partial \rho}+\frac{1}{\rho^{2}} \frac{\partial^{2}}{\partial \varphi^{2}}\right)-\frac{e^{2}}{\epsilon \epsilon_{0} \rho},
$$

where $\rho=(x, y)$ and we have let $z_{e}=z_{h}$ for simplicity. This is nothing but the Hamiltonian of a two-dimensional Hydrogen atom. Its eigenfunctions can be found in analytical form [34]. Here we will be interested in $1 s$ and $2 p$ states. The corresponding wave functions read as

$$
\phi_{X}^{1 s}(\rho)=\sqrt{\frac{2}{\pi\left(2 a_{B}\right)^{2}}} e^{-\rho / 2 a_{B}}
$$

for $1 s$ state and

$$
\begin{aligned}
& \phi_{X}^{2 p \uparrow}(\rho)=\frac{2}{9} \sqrt{\frac{1}{3 \pi\left(2 a_{B}\right)^{2}}} \frac{\rho}{2 a_{B}} e^{-\rho / 6 a_{B}}(\cos \varphi+i \sin \varphi), \\
& \phi_{X}^{2 p \downarrow}(\rho)=\frac{2}{9} \sqrt{\frac{1}{3 \pi\left(2 a_{B}\right)^{2}}} \frac{\rho}{2 a_{B}} e^{-\rho / 6 a_{B}}(\cos \varphi-i \sin \varphi), \\
& \phi_{X}^{2 p}(\rho)=\frac{2}{9} \sqrt{\frac{1}{6 \pi\left(2 a_{B}\right)^{2}}}\left(1-\frac{2}{3} \frac{\rho}{2 a_{B}}\right) e^{-\rho / 6 a_{B}}
\end{aligned}
$$

for $2 p$ states. The latter, in the absence of magnetic field, form a degenerate triplet at the energy $\varepsilon_{2 p}$. This energy can be approximately estimated as the energy of $2 s$ state reported in Ref. [35].

Treating the spontaneously induced electric field $\boldsymbol{E}_{r}$ as a perturbation one can write (in the first order)

$$
\phi_{X}^{1 s p}(\boldsymbol{\rho})=\phi_{X}^{1 s}(\rho)+\sum_{2 p} \frac{\langle 1 s|\hat{V}| 2 p\rangle}{\varepsilon_{2 p}-\varepsilon_{1 s}} \phi_{X}^{2 p}(\boldsymbol{\rho}),
$$

where the summation is over the states (2) and the dipolar interaction operator $\hat{V}$ is given by

$$
\hat{V}=-e \boldsymbol{E}_{r} \cdot \boldsymbol{\rho}=-e E_{r} \rho \cos \varphi .
$$

From Eq. (3) one can see that due to the admixing of $2 p$ states the exciton becomes polarized in the direction of the field $\boldsymbol{E}_{r}$ in the structure plane. Therefore it can now interact with this field and the interaction energy is given by

$$
\varepsilon(r)=\langle 1 s p|\hat{V}| 1 s p\rangle=\sum_{2 p} \frac{|\langle 1 s|\hat{V}| 2 p\rangle|^{2}}{\varepsilon_{2 p}-\varepsilon_{1 s}} .
$$

In general, however, the in-plane electric field can be strong enough to transform the $1 s$ indirect exciton into a strongly mixed hybrid state. The potential energy of such $s p$ hybridized 
exciton can be found by diagonilizing a $3 \times 3$ Hamiltonian,

$$
\hat{H}_{s p}=\left(\begin{array}{ccc}
s & v & v \\
v & p & 0 \\
v & 0 & p
\end{array}\right)
$$

where we have denoted $s=\varepsilon_{1 s}, \quad p=\varepsilon_{2 p}$ and $v=$ $\langle 1 s|\hat{V}| 2 p \uparrow\rangle=\langle 1 s|\hat{V}| 2 p \downarrow\rangle=e E_{r} a_{B} / 2 \sqrt{6}$. One obtains the following set of eigenvalues:

$$
\varepsilon=p,
$$

which corresponds to the $p$-shell oriented perpendicularly to $\boldsymbol{E}_{r}$ and

$$
\varepsilon(r)=\frac{s+p \pm \sqrt{(s-p)^{2}+8 v^{2}(r)}}{2}
$$

for the $s p$ hybrids oriented along $\boldsymbol{E}_{r}$. In what follows, we will be interested in the dependence of the ground-state energy,

$$
\varepsilon_{0}(r)=\frac{\delta-\sqrt{\delta^{2}+8 v^{2}(r)}}{2},
$$

(we have introduced $\delta=p-s$ ) of the polarized indirect exciton on the distance from the center of the ring $r$. Our main goal is thus to calculate the radial electric field $E_{r}$ as a function $r$.

\section{RADIAL DISTRIBUTION OF ELECTRON AND HOLE DENSITIES}

To calculate $E_{r}$, one needs to know the electron and the hole density distributions, $n_{e}$ and $n_{h}$. These can be obtained from the reaction-diffusion model supplemented with the drift term due to the induced electric field. Here we employ the simplest model proposed in Ref. [9], neglecting the interaction with the electric field and allowing the analytic expressions for $n_{e}$ and $n_{h}$. In this model, one neglects the escape of photogenerated holes from the topmost quantum well and assumes that $n_{e}$ and $n_{h}$ are strictly zero inside and outside the ring, respectively. The latter approximation is reasonable for the system studied in Ref. [1], since no significant PL signal is seen in the inner region between the ring and the photoexcitation spot. Kinetic equations for steady state then take the form

$$
\begin{array}{rl}
D_{h} \nabla^{2} n_{h}(r)+P_{x} \delta(\boldsymbol{r})=0 & r \leqslant R, \\
D_{e} \nabla^{2} n_{e}(r)+G-\frac{n_{e}(r)}{\tau_{e}}=0 & r>R .
\end{array}
$$

Here, $D_{e, h}$ are the electron (hole) diffusion constants, $G$ is a spatially uniform source for electrons, $P_{x}$ is the amount of holes injected into the QW per second, $1 / \tau_{e}$ is the electron escape rate due to tunneling. The linear equations (5) can be solved analytically. The relevant length scale of the problem is fixed by the electron depletion length $l=\sqrt{D_{e} \tau_{e}}$. In the limit $R \gg l$, one finds

$$
R=\frac{P_{x}}{2 \pi G l},
$$

i.e., the ring radius depends linearly on the excitation power. For the system under consideration, such behavior of the ring radius has been observed experimentally [15]. This justifies a posteriori the assumption $R \gg l$. In the same limit, the expressions for $n_{e}$ and $n_{h}$ take the form

$$
\begin{aligned}
& n_{h}(x)=p \ln \left(x^{-1}\right) \quad 0<x \leqslant 1, \\
& n_{e}(x)=n\left(1-x^{-1 / 2} e^{-\zeta(x-1)}\right) \quad x>1,
\end{aligned}
$$

where we have introduced $p=P_{x} / 2 \pi D_{h}, n=G \tau_{e}, x \equiv r / R$, and $\zeta=R / l$.

\section{CALCULATION OF THE ELECTRIC FIELD}

The knowledge of the electron and hole density profiles allows one to calculate radial distribution of the electrostatic field $E_{r}$. The further simplification of the model, which one can make here, is to neglect the curvature of the interface. The numerical study shows that this is indeed correct at least for large enough ring radii (compared with the width of the ring) and when one is interested in the field distribution in the vicinity of the interface [the latter corresponds to $x=1$ in (6)].

In this simplified geometry, the total electric field at the point $y \equiv r / R$ can be represented as the superposition of electric fields from a large number of elementary filaments parallel to the interface:

$$
E_{r}(y)=\frac{e}{2 \pi \epsilon \epsilon_{0}}\left(\int^{1} \frac{n_{h}(x) d x}{y-x}-\int_{1} \frac{n_{e}(x) d x}{y-x}\right) .
$$

The calculation of integrals in Eq. (7) is not trivial. The integrals have logarithmic divergencies at the point we are interested in and should be regularized in the sense of the principal value. The resulting set of equations, suitable for numerical calculations, has the form

$$
\begin{aligned}
E_{r}(y)= & \frac{e}{2 \pi \epsilon \epsilon_{0}}\left(\int_{0}^{|y-1|} \frac{ \pm n_{e, h}(y+x) \mp n_{e, h}(y-x)}{x} d x\right. \\
& \left.+\int_{|y-1|}^{x_{0}} \frac{n_{h}(y-x)+n_{e}(y+x)}{x} d x\right)|y-1| \leqslant x_{0} \\
E_{r}(y)= & \frac{e}{2 \pi \epsilon \epsilon_{0}} \int_{0}^{x_{0}} \frac{ \pm n_{e, h}(x+y) \mp n_{e, h}(x-y)}{x} d x \\
& |y-1|>x_{0},
\end{aligned}
$$

where the sign $+/-$ as well as the subscript $e / h$ states for $y>1$ or $y \leqslant 1$, respectively.

Finally, one should bear in mind, that the CQW's are surrounded by conducting contact layers. The contacts will contain electrostatic images of the electron and the hole lakes, located on the distances $2 k h$ from the QW's, where $h$ is the barrier width (we neglect the space between the QW's) and $k=1,2, \ldots$ is an integer. The contribution from the images can be taken into account by replacing

$$
n_{e, h} \longrightarrow n_{e, h}\left(1+2 x \sum_{k=1}^{\infty} \frac{(-1)^{k}}{\sqrt{x^{2}+k^{2} \xi^{2}}}\right)
$$

in the integrals (8), where $\xi \equiv 2 h / R$. 


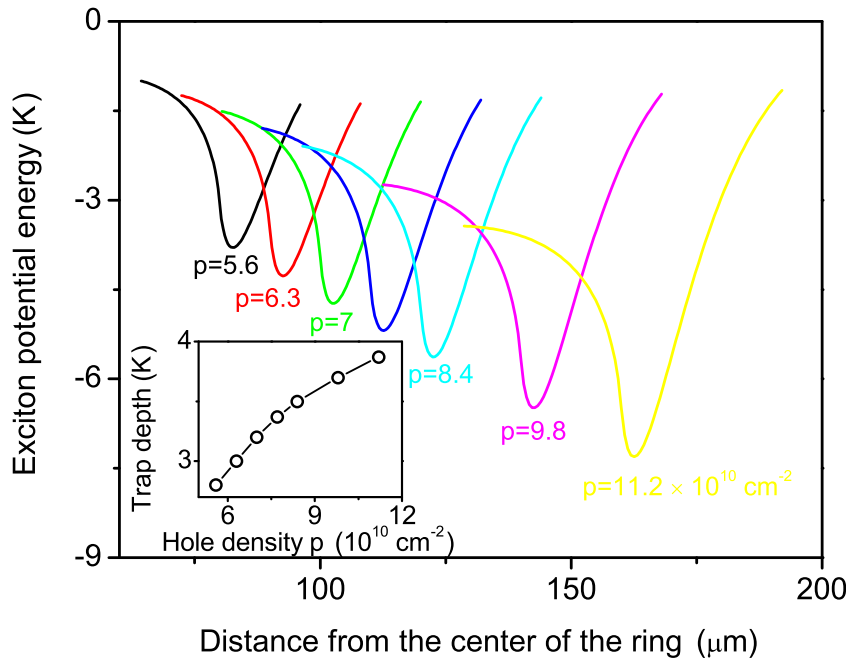

FIG. 2. Built-in electrostatic trap for the radial in-plane motion of indirect excitons at different laser excitation powers. By increasing the excitation power $P$ one injects more holes in the upper QW and, provided that the gate voltage $V_{g}$ is fixed, makes the ring expanding. Assuming that the parameter $p$ depends linearly on the laser power, for the values of parameters chosen one obtains the linear growth of the ring radius $R$ with $P$. At the same time, the depth of the trap increases as is shown in the inset.

\section{RESULTS AND DISCUSSION}

\section{A. External ring}

We first discuss trapping of excitons at the external ring. The exciton potential energy profiles calculated using Eqs. (4) and (8) are presented in Fig. 2. We used the following set of parameters: $n=6 \times 10^{10} \mathrm{~cm}^{-2}, \epsilon=12.9, a_{B}=10 \mathrm{~nm}, h=$ $200 \mathrm{~nm}, l=10 \mu \mathrm{m}$, and $\delta=2 \mathrm{meV}$. The parameter $p$ which governs the radial distribution of holes according to (6) took the values in the range $5.6-11.2 \times 10^{10} \mathrm{~cm}^{-2}$, which corresponds to the increasing laser power $P$. The fixation of the parameter $n$ corresponds to the fixed gate voltage $V_{g}$.

Consistently with the experimental results presented in Refs. [2,15], we assumed the ring radius $R$ being proportional to $p$. For the given value of $p$ the upper bound $x_{0}$ in the integrals (6) had been increased until the shape, the effective width and the height of the trap saturated. This criterion was already achieved at $x_{0} \sim 0.2$, that justifies the simplied geometry we have used. Further increase of $x_{0}$ leads to the shift of the whole picture towards higher energies. The numerical procedure described above qualitatively corresponds to the experimental configuration of Ref. [5] where the PT phase diagram of the external ring was obtained. Remarkably, the depth of the electrostatic trap increases when increasing $\mathrm{P}$ as one can see in the inset of Fig. 2. This observation is in agreement with the excitation power versus the temperature (PT) phase diagram of the external ring reported in Refs. [1,2]. Indeed, the depth of the trap should roughly correspond to the temperature at which the external ring washes out: as soon as the thermal kinetic energy of the excitons exceeds the potential barrier height, the excitons become delocalized.

We point out that even in this simplified model one has a large set of parameters. As expected, the depth of the trap is strongly sensitive to the electron and hole densities. The latter can be estimated from the laser excitation density (see Ref. [12]). The background electron density $n=G \tau_{e}$ can in principle be determined by measuring the electron current through the sample in the absence of photoexcitation [13], provided that the electron escape time from the $\mathrm{QW}$ due to tunneling is known in advance [7]. To the best of our knowledge, neither the electron current nor the tunneling time have not been measured yet. Therefore $n$ was an adjustable parameter of the problem.

\section{B. Linear polarization and coherence textures around an LBS}

An LBS is essentially a ring of a small radius where the electron and the hole density distributions are inverted. As the ring shrinks the excitons form a dense cloud in the center which is seen as a bright spot in the PL pattern. At low temperatures, extended spontaneous coherence and polarization textures have been observed around an LBS. The motion of the excitons forming the coherent state is governed by an effective potential produced by the repulsive interaction with the dense bright core from the internal side and by the electrostatic confinement potential in the outer region. This assumption is consistent with the "mexican-hat" profile of the exciton energy observed in Ref. [3]: the bump in the central part of the hat roughly corresponds to the maximum of the PL intensity. Below we show that the linear polarization and forklike defects in the PL coherence pattern around an LBS can be interpreted as due to spin-orbit coupled BEC of excitons at the ring-shaped trap.

An exciton in GaAs-based quantum wells considered here is composed of an electron with the spin $\pm 1 / 2$ and a heavy hole with the spin $\pm 3 / 2$. Depending on the mutual orientation of the $e$ and $h$ spins, the spin of an exciton may take four possible values:

$$
|X\rangle=\left(\begin{array}{l}
e_{+1 / 2} h_{+3 / 2} \\
e_{+1 / 2} h_{-3 / 2} \\
e_{-1 / 2} h_{+3 / 2} \\
e_{-1 / 2} h_{-3 / 2}
\end{array}\right)=\left(\begin{array}{l}
X_{+2} \\
X_{-1} \\
X_{+1} \\
X_{-2}
\end{array}\right),
$$

where $e_{ \pm 1 / 2}$ and $h_{ \pm 3 / 2}$ stem for the electron and the hole spin components, respectively. As a consequence of the orbital momentum conservation rule, only $X_{-1}$ and $X_{+1}$ components are optically active and contribute to the left $\left(\sigma_{-}\right)$and right $\left(\sigma_{+}\right)$circularly polarized emission of the excitons. The $X_{+2}$ and $X_{-2}$ components are dark.

Earlier theoretical works on spinor condensates (see, for example, Ref. [36]) exploited mainly the idea borrowed from the physics of ferromagnetism: short-range exchange interaction can favor a phase with broken rotational symmetry. The orientation and position of the domain walls in this case are defined by fluctuations of external potential. However, this is not the case for the condensate of indirect excitons. The observed polarization textures are pinned to the crystallographic axes and their orientation is the same for each bright spot.

As was first noticed by A. Kavokin [37], this experimental fact suggests that the exciton spin texture is due to spin-orbit interaction (SOI). Translational motion of an electron and a hole in the electrostatic field of ion cores results in the spin-dependent splitting of the corresponding subbands. In the absence of structural inversion asymmetry (SIA), the 


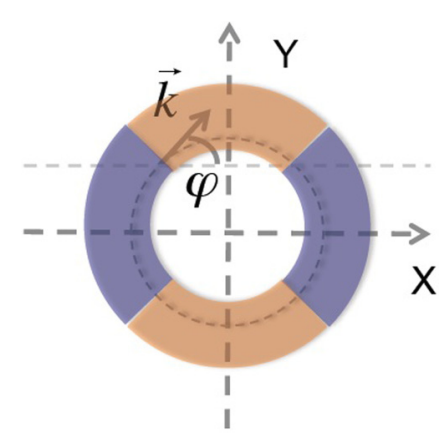

FIG. 3. Schematic illustration of the exciton condensate localized at the ring-shaped channel around an LBS. Color shows the regions with positive (orange) and negative (blue) degrees of linear polarization in a qualitative agreement with the experiment [16]. The emitted light is polarized everywhere along $\mathbf{k}$ according to the result (16).

Hamiltonian of a free exciton takes the form [38]

$\hat{H}_{\mathrm{BIA}}^{X}=\frac{\hbar^{2} k^{2}}{2 m}+\left(\begin{array}{cccc}0 & \beta_{h} k e^{-i \phi} & \beta_{e} k e^{i \phi} & 0 \\ \beta_{h} k e^{i \phi} & 0 & 0 & \beta_{e} k e^{i \phi} \\ \beta_{e} k e^{-i \phi} & 0 & 0 & \beta_{h} k e^{-i \phi} \\ 0 & \beta_{e} k e^{-i \phi} & \beta_{h} k e^{i \phi} & 0\end{array}\right)$,

where $k$ is the absolute value of the exciton wave vector $\boldsymbol{k}$, and $\phi$ is the angle between $\boldsymbol{k}$ and the $x$ axis. The off-diagonal part of (11) can be obtained as a tensor sum $\hat{H}_{\mathrm{BIA}}^{e} \oplus \hat{H}_{\mathrm{BIA}}^{h}$ of an electron [39],

$$
\hat{H}_{\mathrm{BIA}}^{e}=\beta_{e}^{\prime}\left(\sigma_{x} k_{x}^{e}-\sigma_{y} k_{y}^{e}\right),
$$

and a hole [40,41],

$$
\hat{H}_{\mathrm{BIA}}^{h}=\beta_{h}^{\prime}\left(\sigma_{x} k_{x}^{h}+\sigma_{y} k_{y}^{h}\right),
$$

spin Hamiltonians, where one should let $\boldsymbol{k}^{e}=m_{e} \boldsymbol{k} /\left(m_{e}+m_{h}\right)$ and $\boldsymbol{k}^{h}=m_{h} \boldsymbol{k} /\left(m_{e}+m_{h}\right)$ (we implicitly average out the relative motion of an electron and a hole within the exciton). The coordinate axes are chosen as $x \|$ [100] and $y \|$ [010].

The ground state of the Hamiltonian (11) is the plane wave

$$
\left|X_{\phi}\right\rangle=\frac{1}{2} e^{i k_{0} r}\left(\begin{array}{c}
1 \\
-e^{i \phi} \\
-e^{-i \phi} \\
1
\end{array}\right)
$$

with

$$
k_{0}=\frac{m}{\hbar^{2}}\left(\beta_{e}+\beta_{h}\right) .
$$

In our case the local orientation of $\boldsymbol{k}$ is imposed by the ring-shaped confinement, as detailed above and shown schematically in Fig. 3. The macroscopic occupation of the single-particle state (14) results in circulation of excitons around the dense core. The direction of the exciton flow (clockwise or counter-clockwise) is not important for our consideration. By evaluating the intensities

$$
\begin{gathered}
I_{x}=\langle\Psi \mid x\rangle\langle x \mid \Psi\rangle=\frac{1}{2} n(\rho) \cos ^{2} \phi, \\
I_{y}=\langle\Psi \mid y\rangle\langle y \mid \Psi\rangle=\frac{1}{2} n(\rho) \sin ^{2} \phi
\end{gathered}
$$

with $|\Psi\rangle=\sqrt{n(\rho)}\left|X_{\phi}\right\rangle$ and

$$
\begin{aligned}
& \langle x|=(0,1 / \sqrt{2}, 1 / \sqrt{2}, 0) \\
& \langle y|=(0,-i / \sqrt{2}, i / \sqrt{2}, 0),
\end{aligned}
$$

one obtains the linear polarization degree

$$
\rho_{l} \equiv \frac{I_{x}-I_{y}}{I_{x}+I_{y}}=\cos (2 \phi) .
$$

The results (16) and (19) show that the emitted light is polarized everywhere along the condensate velocity $\boldsymbol{v}_{0}=$ $\frac{\hbar}{m} \boldsymbol{k}_{0}$. This is schematically illustrated in Fig. 3. The linear polarization texture (19) corresponds very well to the textures observed around an LBS [16,42].

This way, according to our hypothesis, an LBS can be regarded as a (quantized) vortex of a dilute exciton BEC pinned to a dense core (the origin of the core remains to be understood). Numerical simulations of the shift-interferometry measurement shows that a quantized vortex should be characterized by a pair of opposite forks [14].

\section{CONCLUSIONS}

To conclude, we have shown that the rings of dipolar excitons observed in coupled GaAs quantum wells at low temperatures represent trapped exciton gases. The trapping is due to the electrostatic interaction of the excitons with in-plane electric field induced at the boundary between electron- and hole-rich regions. Our results are in a good agreement with the available experimental data. We suggest that the polarization and coherence patterns observed around LBS can be due to spin-orbit coupled Bose-Einstein condensation of excitons in a ring-shaped trap.

\section{ACKNOWLEDGMENTS}

The author acknowledges M. Petrov for numerical verification of the results and simulation of ring-shaped traps of a small radius. This work has been supported by the Government of the Russian Federation (Grant 074-U01) through ITMO Postdoctoral Fellowship scheme.
[1] L. V. Butov, A. C. Gossard, and D. S. Chemla, Nature (London) 418, 751 (2002).

[2] D. Snoke, S. Denev, Y. Liu, L. Pfeiffer, and K. West, Nature (London) 418, 754 (2002).
[3] L. V. Butov, C. W. Lai, A. L. Ivanov, A. C. Gossard, and D. S. Chemla, Nature (London) 417, 47 (2002).

[4] C. W. Lai, J. Zoch, A. C. Gossard, and D. S. Chemla, Science 303, 503 (2004). 
[5] L. V. Butov, L. S. Levitov, A. V. Mintsev, B. D. Simons, A. C. Gossard, and D. S. Chemla, Phys. Rev. Lett. 92, 117404 (2004).

[6] R. Rapaport, G. Chen, D. Snoke, S. H. Simon, L. Pfeiffer, K. West, Y. Liu, and S. Denev, Phys. Rev. Lett. 92, 117405 (2004).

[7] S. Yang, L. V. Butov, L. S. Levitov, B. D. Simons, and A. C. Gossard, Phys. Rev. B 81, 115320 (2010).

[8] M. Alloing, M. Beian, M. Lewenstein, D. Fuster, Y. Gonzalez, L. Gonzalez, R. Combescot, M. Combescot, and F. Dubin, Europhys. Lett. 107, 10012 (2014).

[9] M. Haque, Phys. Rev. E 73, 066207 (2006).

[10] L. Mouchliadis and A. L. Ivanov, J. Phys.: Condens. Matter 19, 295215 (2007).

[11] J. Wilkes, E. A. Muljarov, and A. L. Ivanov, Phys. Rev. Lett. 109, 187402 (2012).

[12] To be more precise, the lake of a positive charge is formed by an excess of holes, since the photoexcited electrons are also present in the opposite QW, though in lower number. Binding and recombination of these electrons with the holes gives rise to a smaller PL ring in the immediate vicinity of the photoexcitation spot, see, e.g., M. Remeika, J. C. Graves, A. T. Hammack, A. D. Meyertholen, M. M. Fogler, L. V. Butov, M. Hanson, and A. C. Gossard, Phys. Rev. Lett. 102, 186803 (2009). This inner ring has subsequently been observed in the bulk GaAs as well [S. Bieker, T. Henn, T. Kiessling, W. Ossau, and L. W. Molenkamp, Phys. Rev. B 90, 201305(R) (2014); Phys. Rev. Lett. 114, 227402 (2015)] and has been shown to result from a temperature gradient within the exciton gas.

[13] S. Yang, A. T. Hammack, M. M. Fogler, L. V. Butov, and A. C. Gossard, Phys. Rev. Lett. 97, 187402 (2006).

[14] A. A. High, J. R. Leonard, A. T. Hammack, M. M. Fogler, L. V. Butov, A. V. Kavokin, K. L. Campman, and A. C. Gossard, Nature (London) 483, 584 (2012).

[15] S. V. Andreev, Phys. Rev. Lett. 110, 146401 (2013).

[16] A. A. High, A. T. Hammack, J. R. Leonard, Sen Yang, L. V. Butov, T. Ostatnicky, M. Vladimirova, A. V. Kavokin, T. C. H. Liew, K. L. Campman, and A. C. Gossard, Phys. Rev. Lett. 110, 246403 (2013).

[17] A. V. Kavokin, M. Vladimirova, B. Jouault, T. C. H. Liew, J. R. Leonard, and L. V. Butov, Phys. Rev. B 88, 195309 (2013).

[18] S. V. Andreev, Phys. Rev. B 92, 041117(R) (2015); 94, 140501(R) (2016).

[19] D. V. Vishnevsky, H. Flayac, A. V. Nalitov, D. D. Solnyshkov, N. A. Gippius, and G. Malpuech, Phys. Rev. Lett. 110, 246404 (2013).

[20] L. V. Butov, JETP 122, 434 (2016).

[21] S. V. Andreev, A. A. Varlamov, and A. V. Kavokin, Phys. Rev. Lett. 112, 036401 (2014).

[22] In the system under consideration the constant chemical potential is achieved due to a dynamical equilibrium between the localized excitons and delocalized electron-hole plasma.

[23] G. J. Schinner, E. Schubert, M. P. Stallhofer, J. P. Kotthaus, D. Schuh, A. K. Rai, D. Reuter, A. D. Wieck, and A. O. Govorov, Phys. Rev. B 83, 165308 (2011).
[24] D. P. Trauernicht, A. Mysyrowicz, and J. P. Wolfe, Phys. Rev. B 28, 3590 (1983).

[25] K. Kash, J. M. Worlock, M. D. Sturge, P. Grabbe, J. P. Harbison, A. Scherer, and P. S. D. Lin, Appl. Phys. Lett. 53, 782 (1988).

[26] V. Negoita, D. W. Snoke, and K. Eberl, Appl. Phys. Lett. 75, 2059 (1999).

[27] K. Brunner, U. Bockelmann, G. Abstreiter, M. Walther, G. Böhm, G. Tränkle, and G. Weimann, Phys. Rev. Lett. 69, 3216 (1992).

[28] P. C. M. Christianen, F. Piazza, J. G. S. Lok, J. C. Maan, and W. van der Vleuten, Physica B 249, 624 (1998).

[29] A. T. Hammack, M. Griswold, L. V. Butov, L. E. Smallwood, A. L. Ivanov, and A. C. Gossard, Phys. Rev. Lett. 96, 227402 (2006).

[30] A. A. High, J. R. Leonard, M. Remeika, L. V. Butov, M. Hanson, and A. C. Gossard, Nano Lett. 12, 2605 (2012).

[31] A. T. Hammack, N. A. Gippius, Sen Yang, G. O. Andreev, L. V. Butov, M. Hanson, and A. C. Gossard, J. Appl. Phys. 99, 066104 (2006).

[32] A. V. Gorbunov and V. B. Timofeev, JETP Lett. 84, 329 (2006).

[33] R. Rapaport, G. Chen, S. Simon, O. Mitrofanov, L. Pfeiffer, and P. M. Platzman, Phys. Rev. B 72, 075428 (2005).

[34] X. L. Yang, S. H. Guo, F. T. Chan, K. W. Wong, and W. Y. Ching, Phys. Rev. A 43, 1186 (1991).

[35] K. Sivalertporn, L. Mouchliadis, A. L. Ivanov, R. Philp, and E. A. Muljarov, Phys. Rev. B 85, 045207 (2012).

[36] J. Stenger, S. Inouye, D. M. Stamper-Kurn, H.-J. Miesner, A. P. Chikkatur, and W. Ketterle, Nature (London) 396, 345 (1998).

[37] Private discussion at the International School on Nanophotonics and Photovoltaics in Tsakhkadzor (Armenia) (2010).

[38] Noteworthy, the Hamiltonian (11) was used in the previous theoretical model $[16,17]$. In contrast to our model, the authors assumed radial propagation of excitons from the source. No assumption of the macroscopic occupation of the ground state of (11) has been made. Good agreement with the experimental data was achieved by a proper choice of the boundary (initial) conditions in a numerical simulation.

[39] M. I. Dyakonov and V. Yu. Kachorovskii, Fiz. Tekh. Poluprovodn. 20, 178 (1986) [Sov. Phys. Semicond. 20, 110 (1986)].

[40] M. V. Durnev, M. M. Glazov, and E. L. Ivchenko, Phys. Rev. B 89, 075430 (2014).

[41] Note that the authors of Ref. [40] used an inverted order of the basic states at the $\Gamma$ point in the heavy-hole subband.

[42] Weak intensity of the light in the region where the extended coherence was observed can be related to the fact, that for the typical values of parameters that the wave vector of the condensate $\boldsymbol{k}_{0}$ slightly exceeds $k_{\max } \sim 26 \mu \mathrm{m}^{-1}$ defined by the light cone. Also one can see from (16) that only half of the total number of excitons is effectively in the bright state. Half of the condensate is dark. 